\title{
Mit vorwärtsorientierter Politik gegen rückwärtsgerichtete Xenophobie
}

\author{
COLIN CROUCH
}

$\mathrm{E}$ s gibt Menschen, die die Begegnungen mit anderen Kulturen scheuen. Und es gibt Politiker, die bereit stehen, dieses Unbehagen auszubeuten. In der Vergangenheit hat sich ein solcher Politkertypus jedoch eher bedeckt gehalten; nicht zuletzt, weil aufgeklärte und demokratische Gesellschaften der Nachkriegszeit Fremdenfeindlichkeit (Xenophobie) aus gutem Grund und geschichtlicher Erfahrung nicht toleriert haben. Heute aber stehen wir vor einem Wendepunkt: Nicht nur die Scham, fremdenfeindliche Positionen zu beziehen, verflüchtigt sich, sondern die Ausgrenzung des scheinbar „Fremden“ greift in aggressiver Weise um sich. Die Erklärungsansätze für diese Entwicklung sind bekannt: es sei die Unsicherheit gegenüber den Folgen der Globalisierung, die Menschen um- und in nationale Abschottungen hineintreibt; es sei die Angst vor Einwanderung und die damit verbundene Begegnung mit fremden Kulturen. Und es sei die Furcht, dass terroristische Bedrohungen anwachsen könnten.

Derartige Ängste haben bereits politische Wirkung entfaltet. Beispiele sind: Die Entscheidung einer Mehrheit der britischen Wähler, die Europäische Union zu verlassen; die Wahl Trumps zum US-Präsidenten; die xenophobe Wende der österreichischen, dänischen, ungarischen, polnischen Politik (die Aufzählung wäre fortzusetzen) und speziell in Deutschland das Erstarken der xenophoben Partei Alternative für Deutschland. Jedes dieser Ereignisse steht dafür, dass fremdenfeindliche Gefühle und Äußerungen öffentlich werden. Mehr noch: Sozialliberale Meinungen und insbesondere diejenigen, die den (immer noch mehrheitlichen!) gesellschaftlichen Konsens vertreten, sich gegen Xenophobie zu stemmen, werden als angeblich von den Nöten und vom Willen der „normalen Leute" abgekoppelte politische und (groß)städtische Eliten beschimpft.

Für so manchen liberalen und sozialdemokratischen Politiker gibt es eine große Versuchung, der fremdenfeindlichen Wende zu folgen - sei es, um der als Schimpfwort gemeinten Zuschreibung zu einer „Elite“ zu entgehen, sei es aus der Hoffnung, dass kleine Zugeständnisse schlimmere Folgen vermeiden und abgewanderte Wähler zurückgewinnen könnten. Jedoch: Kompromissstrategien verschieben nichtdisponible Grenzen, die jeden Einzelnen und uns als Gesellschaft vor Fremdenfeindlichkeit schützen. Jedes Mal, wenn eine prominente Person xenophobe Meinungen äußert, bewegen sich die kulturellen und moralischen Grenzen der Gesellschaft ein Stück weiter in eine fremdenfeindliche Richtung. Wir sollten uns stattdessen darüber bewusst sein: Eine liberale Grundeinstellung darf nicht angetastet werden, denn sie ist die beste Waffe gegen Hass und Intoleranz.

Das Festhalten an einem liberalen Politikverständnis schließt allerdings nicht aus, Entscheidungen der jüngeren Vergangenheit nochmals kritisch zu reflektieren: Dies betrifft die Anzahl der Einwanderer und die Verteilung der Geflüchteten. Menschen, die in großen Städten leben und somit auch Erfahrungen mit Zuwanderung diverser Nationalitäten haben, sind normalerweise liberaler eingestellt als Menschen in Regionen oder kleinen Städten, in denen erstmals größere Gruppen von Einwanderern ankommen. Rückblickend war es ggf. ein Fehler, dass die britische Regierung bereits Jahre vor den meisten anderen EU-Mitgliedern entschieden hat, Einwanderern aus Polen und anderen osteuropäischen Ländern freien Zutritt nach Großbritannien zu gewähren. Dies hat dor zur „Brexit-Stimmung" beigetragen. Und es war politisch gesehen wahrscheinlich auch ein Fehler, als Deutschland seine Grenzen für den Zustrom von Geflüchteten aus dem mittleren Osten spontan geöffnet hat - obwohl diese Entscheidung das moralisch beste Beispiel für Großzügigkeit und Menschlichkeit war, das ein westliches Land gegenüber den Opfern der Kriege in und der Vertreibungen aus diesen Regionen gezeigt hat.

Menschen, die in prosperierenden Regionen leben, die mit ihrem Bezirk oder ihrer Stadt positiv verbunden sind und die Erfahrungen mit Immigration haben, haben weniger Angst vor der Globalisierung und einer kosmopolitischen Zukunft. Eine geografische Analyse der Wahlen in Großbritannien, den USA, Frankreich, Deutschland, Österreich und anderen Ländern zeigt, dass sich Xenophobie dort bündelt, wo es an einer solchen regionalen Verwurzelung und Zufriedenheit fehlt. Doch es gibt auch - und das wird in den politischen Diskussionen vielfach vergessen - wohlhabende, konservative Gemeinschaften, die einen guten Lebensstandard, aber gleichwohl Abstiegsängste haben. Diese zwei Gruppen - Menschen in weniger prosperierenden Regionen und Menschen in wohlhabenden konservativen Milieus - haben zwar unterschiedliche Einkommensniveaus und Lebensstile. Was sie eint, ist ihre Unzufriedenheit mit einer modernen, sich globalisierenden, rasch verändernden Welt und folglich eine tiefe Sehnsucht nach der Vergangenheit. Sie stimmen für „ein besseres Gestern“, für eine kleine Welt, die gegen feindliche externe Mächte gewappnet ist, die weder Globalisierung noch Kosmopolitismus kennt. Und hier liegt der Nährboden xenophober Einstellungen.

Jedoch: Es gibt keinen Weg zurück „nach Gestern“. Wir brauchen eine vorwärtsorientierte Politik. Es sollte eine vordringliche Aufgabe der Politik sein, den Menschen ein würdiges Auskommen zu ermöglichen, in Städte und Regionen zu investieren, Lebensbedingungen zu schaffen, in denen Menschen ihre Heimat in der globalisierten Wirtschaft finden. Zufriedenheit ist eine notwendige Bedingung für eine offenherzige Bevölkerung. Die Lösung für unser zeitgenössisches Unwohlsein liegt in der Förderung nachhaltiger lokaler wirtschaftlicher Entwicklung. Das meint weit mehr als Logistikdienstleistungen und Call-Center: Es geht um Tätigkeiten, die gebildeten jungen Menschen adäquate Perspektiven bieten.

Natürlich ist auch dieser Weg kein Allheilmittel: Rechts-konservative Milieus wird man auch so nicht erreichen. Aber je mehr wir in zukunftsorientierte, dynamische wirtschaftliche Tätigkeiten investieren, umso geringer wird die Anziehungskraft von Fremdenfeindlichkeit und des Rechtspopulismus.

COLIN CROUCH ist auswärtiges wissenschaftliches Mitglied des Max-Planck-Instituts für Gesellschaftsforschung (MPI) zu Köln und Professor em. an der Universität von Warwick/Großbritannien. Forschungsschwerpunkte: Demokratie und Soziologie der europäischen Gesellschaften.

colincrouch@me.com 